\title{
High-enthalpy crystalline phases of cadmium telluride
}

\author{
Adebayo O. Adeniyi, ${ }^{1}$ Martin Kunz $\odot,{ }^{2}$ Elissaios Stavrou $\odot,{ }^{3, *}$ and Yansun $\mathrm{Yao}^{1, \dagger}$ \\ ${ }^{1}$ Department of Physics and Engineering Physics, University of Saskatchewan, Saskatoon, Saskatchewan, Canada S7N 5E2 \\ ${ }^{2}$ Advanced Light Source, Lawrence Berkeley Laboratory, Berkeley, California 94720, USA \\ ${ }^{3}$ Physical and Life Sciences Directorate, Lawrence Livermore National Laboratory, Livermore, California 94550, USA
}

(Received 17 January 2020; revised 6 April 2020; accepted 24 June 2020; published 14 July 2020)

\begin{abstract}
Cadmium telluride (CdTe) has been studied using synchrotron powder x-ray diffraction in a diamond anvil cell up to $63 \mathrm{GPa}$ at room temperature combined with density functional calculations. The experiment reveals two pressure-induced structural phase transitions with the following sequence: rocksalt $\rightarrow \mathrm{Cmcm}$ (12 GPa) $\rightarrow$ post- $\mathrm{Cmcm}$ (42 GPa). The crystal structure of the long-sought post- $\mathrm{Cmcm}$ phase is characterized. It has a monoclinic unit cell and the $P 2_{1} / m$ space group. Calculation confirms this phase transition sequence and further predicts a $P 2_{1} / m$ to $P 4 / \mathrm{nmm}$ transition near $68 \mathrm{GPa}$. Interestingly, the enthalpy of CdTe is found to be higher than the enthalpy sum of its constituents $\mathrm{Cd}$ and Te at pressures higher than $34 \mathrm{GPa}$. This suggests that CdTe becomes a high-enthalpy compound at high pressure. Extraordinary dynamic stability revealed in the phonon dispersion relations prevents the decomposition of CdTe at high pressure.
\end{abstract}

DOI: 10.1103/PhysRevResearch.2.033072

\section{INTRODUCTION}

Cadmium telluride (CdTe) is a direct-band-gap semiconductor with a band-gap size matching almost perfectly to the solar spectrum [1,2]. This trait makes CdTe an optimal material for low-cost high-efficiency photovoltaic applications. Current state-of-the-art CdTe-based single-junction devices can reach a conversion efficiency as high as $22 \%$, providing a low-cost alternative to conventional silicon-based devices [3]. Moreover, doped CdTe and variants have numerous applications in thermoelectrics, ferroelectrics, and quantum dots [4-6]. Driven by its versatile applications, the study of $\mathrm{CdTe}$ is currently very active. Since the properties of CdTe are closely tied to its crystal structure, understanding the latter and its changes under external stimuli is a key step to developing new applications.

At ambient conditions, crystalline CdTe adopts the semiconducting zinc-blende structure, which is a common structure for group II-VI binary compounds. Near 3.5 GPa, CdTe transforms into a semimetallic rocksalt structure via an intermediate cinnabar structure which has a very narrow region of stability [7,8]. A further transition occurs at $10 \mathrm{GPa}$ to a metallic $\mathrm{Cmcm}$ structure [8]. The formation of a metallic phase in the $\mathrm{Cmcm}$ structure is attributed to the distortion of the structure driven by soft-phonon modes in the transverse acoustic branch [9]. A further transition of CdTe was

\footnotetext{
*stavrou1@1lnl.gov

†yansun.yao@usask.ca

Published by the American Physical Society under the terms of the Creative Commons Attribution 4.0 International license. Further distribution of this work must maintain attribution to the author(s) and the published article's title, journal citation, and DOI.
}

observed at $42 \mathrm{GPa}$ to an unidentified phase (the post- $\mathrm{Cmcm}$ phase) [10]. Until now, little is known about this phase, except it is stable to at least $55 \mathrm{GPa}$. Theoretically, the zinc-blende $\rightarrow$ cinnabar $\rightarrow$ rocksalt $\rightarrow \mathrm{Cmcm}$ transition sequence and critical pressures were accurately reproduced using density functional theory (DFT)-based calculations $[9,11]$. A phase transition had been predicted at $44 \mathrm{GPa}$ [9], which is in fact very close to the experimental value, but the predicted structure $(P-3 m 1)$ clearly does not match the x-ray-diffraction (XRD) pattern of the post-Cmcm phase. Moreover, DFT calculation shows that the enthalpy of CdTe should become higher than that of its elemental constituents $(\mathrm{Cd}$ and $\mathrm{Te})$ at $34 \mathrm{GPa}$, indicating that CdTe may actually undergo an elemental dissociation in this pressure neighborhood [9].

To date, the post- $\mathrm{Cmcm}$ phase of CdTe remains unknown and the predicted dissociation in this pressure neighborhood adds to the mystery. To address this problem and understand the mechanisms underlying the interesting phase transitions, we carried out a detailed synchrotron XRD and computational study of CdTe in the rocksalt to post- $\mathrm{Cmcm}$ regions (6$63 \mathrm{GPa}$ ). Our experiment successfully reproduces the rocksalt to $\mathrm{Cmcm}$ transition at $10.5 \mathrm{GPa}$ and the $\mathrm{Cmcm}$ to post- $\mathrm{Cmcm}$ transition at $42 \mathrm{GPa}$. The measured XRD pattern for the post$\mathrm{Cmcm}$ phase agrees very well with the previously reported pattern. Using the metadynamics method and Le Bail refinement, we identify the structure of this phase as a monoclinic $P 2_{1} / m$ structure. The $P 2_{1} / m$ structure is a distorted form of the $B 11$ structure $(P 4 / \mathrm{nmm})$ and can be viewed as an intermediate phase for the $\mathrm{Cmcm}$ to $B 11$ transition in a broader pressure range. Consistent with previous reports, both $P 2_{1} / m$ and $B 11$ structures are found to have higher enthalpies than their elemental constituents. This extraordinary phenomenon is attributed to a significant kinetic energy barrier separating crystalline CdTe from elemental phases in composition space. 


\section{METHODS}

\section{A. Experimental methods}

High-purity commercially available (Sigma-Aldrich greater than $99.9999 \%$ ) CdTe was grounded to fine powder for the angle dispersive XRD measurements. The sample and pressure sensors (gold and ruby) were loaded into a diamond-anvil cell with neon as a pressure transmitting medium (PTM). A Mar-CCD detector was used at the Advanced Light Source, Lawrence Berkeley National Laboratory Beamline 12.2.2 to collect pressure-dependent XRD data. The $\mathrm{x}$-ray probing beam spot size was focused to approximately $10 \mu \mathrm{m}$. Additional details on the XRD experimental setups are given in Ref. [12]. Pressure was determined using a known ambient temperature equation of state (EOS) of gold [13] and also calibrated ruby luminescence [14]. Integration of powder-diffraction patterns to yield scattering intensity versus $2 \theta$ diagrams and initial analysis were performed using the DIOPTAS program [15]. Calculated XRD patterns were produced using the POWDER CELL program [16] for the corresponding crystal structures according to the EOSs determined experimentally and theoretically in this study and assuming continuous Debye rings of uniform intensity. Le Bail refinements were performed using the GSAS software [17]. Indexing of XRD patterns has been performed using the DICVOL program [18] as implemented in the FULLPROF suite.

\section{B. Computational methods}

First-principles metadynamics calculations [19] were performed to simulate the high-pressure phase transitions of $\mathrm{CdTe}$ and identify different structures. The simulation has been carried out using the projector augmented plane-wave (PAW) potentials $[20,21]$ and the Vienna ab initio simulation (VASP) program [22]. The PAW potentials with the PerdewBurke-Ernzerhof functional [23] and a 650-eV energy cutoff were used for $\mathrm{Cd}$ and $\mathrm{Te}$, which employ $4 s^{2} 4 p^{6} 4 d^{10} 5 s^{2}$ and $5 s^{2} 5 p^{4}$ as valence states, respectively. The $\mathrm{Cmcm}$ structure was used as the starting structure for the simulation at room temperature $(300 \mathrm{~K})$ and in the pressure range of 50-120 GPa, using various supercells that consisted of 8-32 CdTe formula units (f.u.) along with a $k$ spacing of $2 \pi \times 0.08 \AA^{-1}$ for the Brillouin zone sampling. Each metastep consisted of a molecular dynamics simulation within the canonical $(N V T)$ ensemble for a simulation time of 0.8 ps. Enthalpy and electronic structure calculations for candidate structures were conducted using the same PAW potentials and VASP but with a fine $k$ spacing of $2 \pi \times 0.03 \AA^{-1}$. Phonon calculations were performed using the quantum ESPRESSO package [24] with norm-conserving pseudopotentials and an energy cutoff of 75 Rydberg, along with a $4 \times 4 \times 4 q$-point mesh and an $8 \times 8 \times 8 k$-point mesh.

\section{RESULTS AND DISCUSSION}

The XRD patterns of the CdTe sample measured at several pressures on pressure increase clearly revealed a series of phase transitions (Fig. 1). At low pressure (6-10.5 GPa), the XRD patterns can be unambiguously indexed to the rocksalt

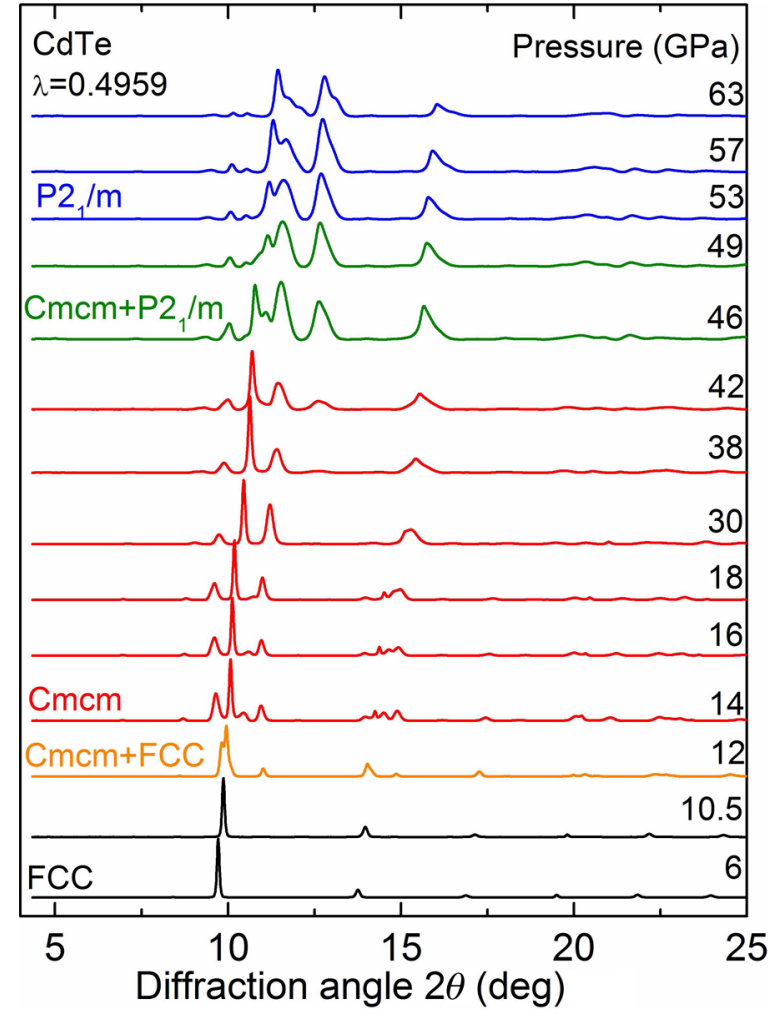

FIG. 1. Experimental XRD patterns of CdTe at various pressures measured on pressure increase. The XRD patterns of the rocksalt, $\mathrm{Cmcm}$, and post- $\mathrm{Cmcm}$ phases are shown by black, red, and blue lines, respectively. The XRD patterns of mixtures of $\mathrm{Cmcm}$ and rocksalt and of $\mathrm{Cmcm}$ and post-Cmcm $\left(P 2_{1} / \mathrm{m}\right)$ phases are shown by yellow and green lines, respectively.

structure. The $d$ spacings of the observed peaks match perfectly a face-centered-cubic lattice. The strongest reflection peak at around $10^{\circ}$ is from the (200) reflection, while the other five peaks are (220), (222), (400), (420), and (422). Since Cd and Te have close number of electrons, the (111) reflection by the two elements almost cancels out and therefore appears to be absent. At $12 \mathrm{GPa}$, the first phase transition takes place, shown by the appearance of additional Bragg peaks. Moreover, the (200) peak splits into three peaks, namely, (020), (200), and (002), corresponding to an orthorhombic distortion. Indexing of the whole XRD pattern revealed that the crystal structure is indeed the $\mathrm{Cmcm}$ as previously reported. The $\mathrm{Cmcm}$ structure is observed to be stable until at least $42 \mathrm{GPa}$ on pressure increase.

The pressure dependence of the lattice parameters and volumes for the rocksalt and $\mathrm{Cmcm}$ structures from 6 to $46 \mathrm{GPa}$ were obtained from the measured $d$ spacings and compared with the calculated values [Figs. 2(a) and 2(b)]. The onset of the phase transition appears near $12 \mathrm{GPa}$. In the transition region, all three lattice parameters undergo continuous changes with no obvious discontinuity in unit cell volume, in agreement with a previous study [8]. The changes, which are the $a$-axis decreasing at a rate similar to that for the rocksalt structure and the $b$-axis increasing and $c$-axis decreasing at much higher rates, reveal an evolution of the cubic lattice to an orthorhombic lattice. This transition is therefore dictated 

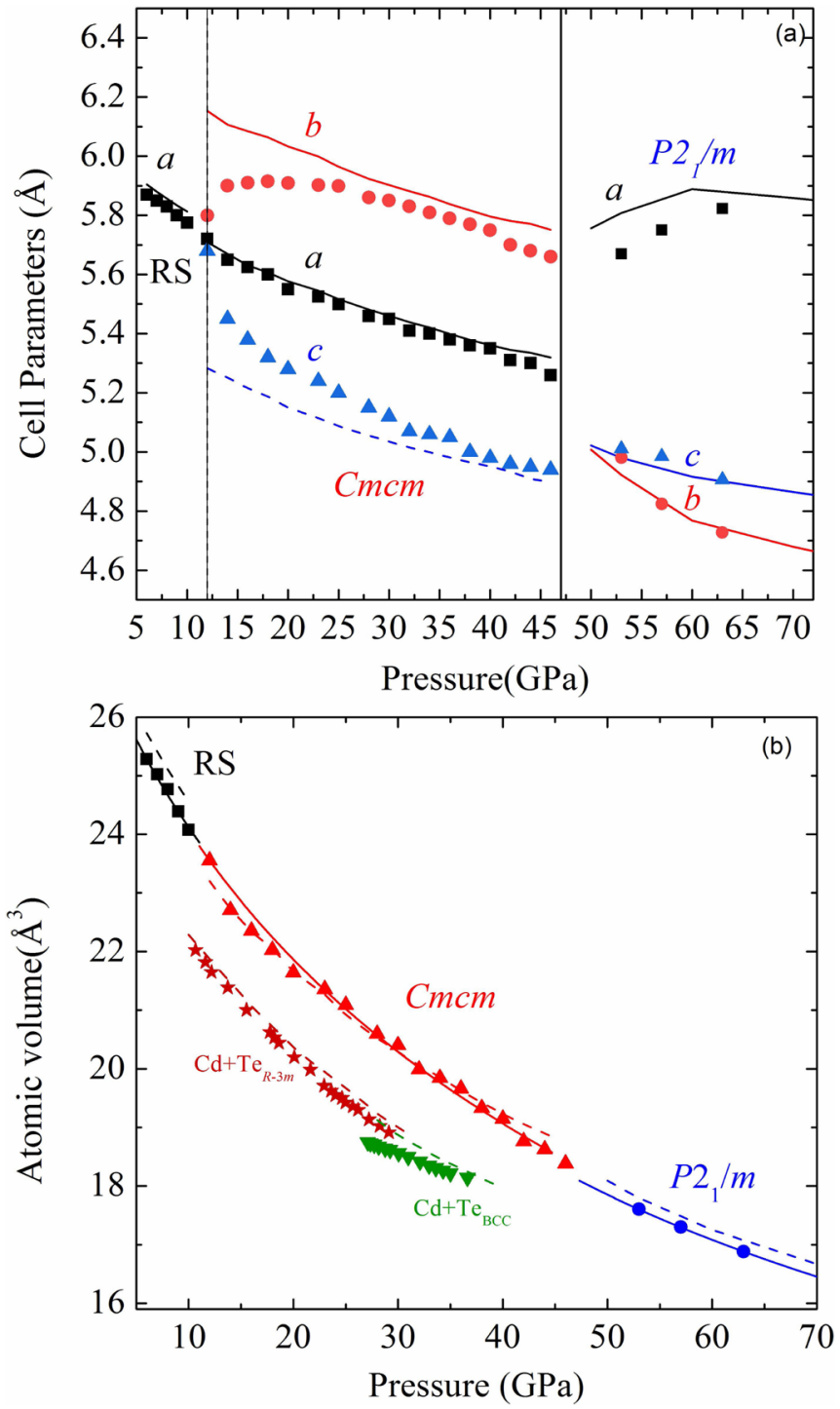

FIG. 2. (a) Pressure dependence of the lattice parameters of $\mathrm{CdTe}$ and (b) volume-pressure data for the rocksalt, $\mathrm{Cmcm}$, and post-Cmcm CdTe. Experimental and calculated values are shown with solid symbols and dashed lines, respectively. The solid lines in (b) are unweighted third-order Birch-Murnaghan EOS fits to the experimental data points [25]. In (b) the experimental [26,27] and the calculated volume of the superposition of $(\mathrm{Cd}+\mathrm{Te}) / 2$ are also shown for comparison.

by a rectangular distortion of the (001) planes along with relative shearing of the neighboring planes along in the [010] direction. During the shearing, the interplanar distance ( $c$ axis) has to decrease at a higher rate to accommodate the volume change.

This transition mechanism is visualized in Fig. 3. In this transition, the sixfold coordination of the rocksalt structure evolves to $5+3$ coordination in the $\mathrm{Cmcm}$ structure. Once the $\mathrm{Cmcm}$ structure is fully settled (above $30 \mathrm{GPa}$ ), the three lattice parameters decrease all together at about the same rate. The calculation matches very well the experiment for the rocksalt structure and the $\mathrm{Cmcm}$ structure at the highpressure end [Fig. 2(a)]. In the transition region, however, the
DFT calculation was not able to reproduce the continuous nature of the transition, instead showing a sudden jump at the transition point. From an experimental perspective it is possible that there are significant internal strains in the sample which can hold the orthorhombic structure at intermediate stages, resulting in a large hysteresis for the phase transition and the coexistence of two phases. The structure optimization calculation, on the other hand, has no constraints on degrees of freedom and therefore can only be settled on the ending structure. The interesting changes of lattice parameters across the rocksalt to $\mathrm{Cmcm}$ transition has been observed previously, which caused confusion and led to incorrect identification of the $C m c m$ structure $[28,29]$. We conducted unweighted fits to the experimental $P-V$ data using a third-order BirchMurnaghan EOS [25] and determined the bulk modulus $B$ and its first derivative $B^{\prime}$ at the experimental onset pressure for the rocksalt and the $C \mathrm{mcm}$. The elastic parameters obtained in this way are given in Table I.

The onset of the second phase transition was observed at $46 \mathrm{GPa}$ (Fig. 1). This is also a continuous transition that spans over a course of $7 \mathrm{GPa}$. The obtained phase is stable to at least $63 \mathrm{GPa}$, the highest pressure attained in this study. The XRD pattern for the phase (i.e., at $53 \mathrm{GPa}$ ) bears a close resemblance to that of the previously reported post- $\mathrm{Cmcm}$ phase [10]. A careful inspection of the XRD pattern shows that this structure does not belong to any known structures of CdTe and other group II-VI binary compounds. A preliminary indexing of the XRD pattern at $63 \mathrm{GPa}$ shows that it has a $P$-type monoclinic cell with $Z=4$ (f.u. per unit cell). However, this solution is inconclusive without the knowledge of atomic positions in the unit cell. To address the structure, metadynamics calculations were performed to simulate phase transitions starting from the $\mathrm{Cmcm}$ structure. The simulation was carried out in a vast pressure region, from 50 to $120 \mathrm{GPa}$, to capture as many phase transitions as our computation resource can afford. Many structures were found to have competitive enthalpies in this region but with different space groups, i.e., $P-1, C 2 / m, C 2 / c, P 2_{1} / m, P 2 / m, P 2_{1} / c, P b c m$, $I 4 / \mathrm{mmm}$, and $P-3 \mathrm{~m} 1$. Out of all structures, the one that matches best the experimental XRD pattern of the post- $\mathrm{Cmcm}$ phase is the $P 2_{1} / m$ structure with $Z=4$.

Figure 4(a) shows a comparison of the simulated XRD pattern for the $P 2_{1} / m$ structure to the experimental pattern at $63 \mathrm{GPa}$. It can be seen that all major reflection peaks and features are well reproduced by this structure. At high $2 \theta$ angles (greater than $20^{\circ}$ ), the experimental XRD pattern is degraded because of the reduced diffracted intensity, but the calculation nevertheless reveals several reflections. The experimental XRD pattern is then analyzed by performing Le Bail refinements in order to determine the structural parameters. Preferred orientation effects and strongly anisotropic peak broadening effects, which are usual under high-pressure conditions, prevent a full structural refinement (Rietveld) of the positional parameters. Thus, the theoretically determined positional parameters are used as starting parameters. A typical refined high-pressure pattern is shown in Fig. 4(b). The corresponding structural parameters along with the theoretical values are summarized in Table I. In view of this good match, we suggest the $P 2_{1} / m$ structure as the structure of the postCmcm phase. 


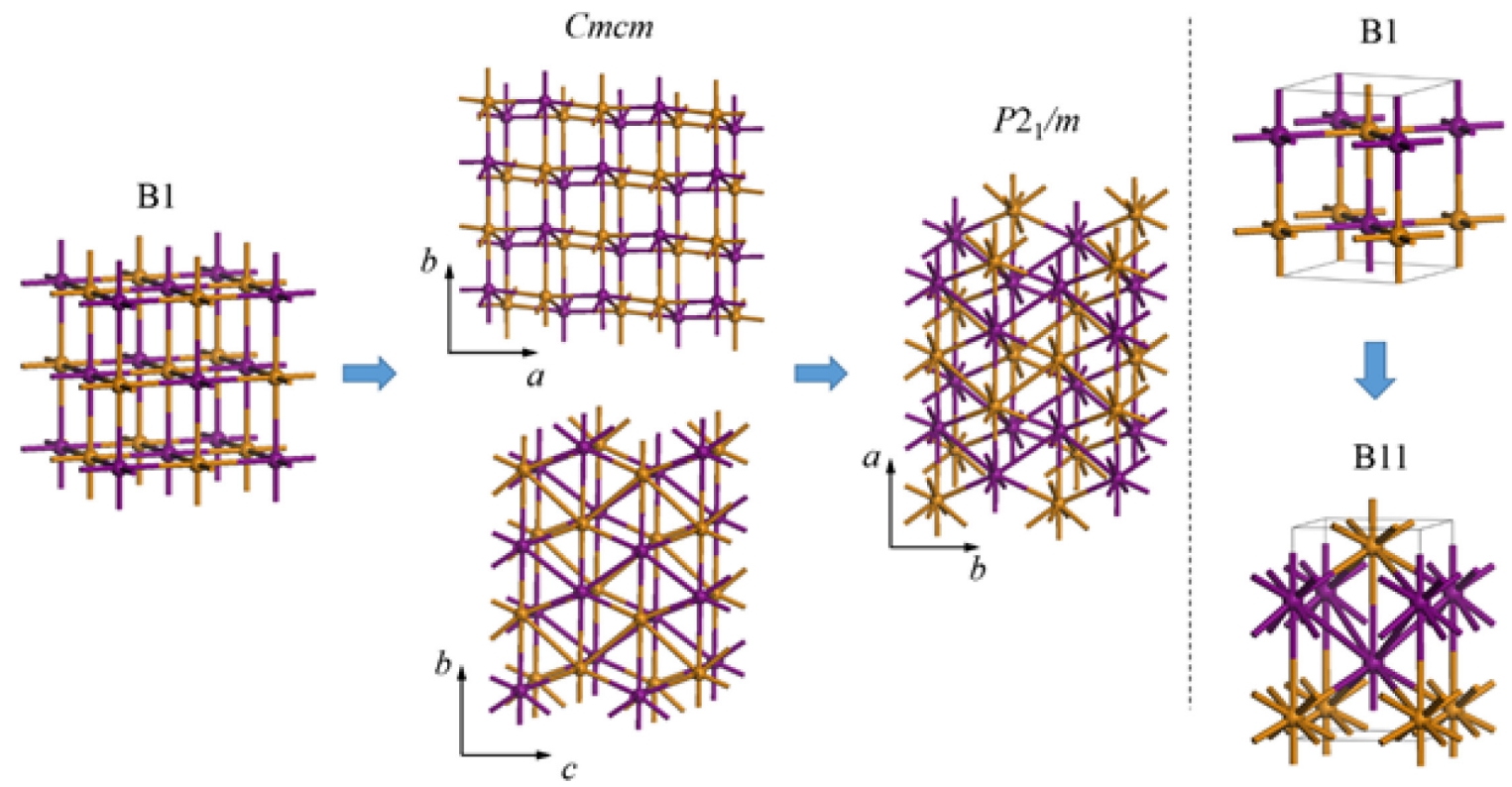

FIG. 3. Transition pathway for the $B 1 \rightarrow C m c m \rightarrow P 2_{1} / m$ phase transition. Shown on the right is the structural relation between $B 1$ and $B 11$ structures. The $\mathrm{Cd}$ and $\mathrm{Te}$ atoms are colored purple and yellow, respectively.

From the XRD data, the lattice parameters and the cell volume of the $P 2_{1} / \mathrm{m}$ structure are determined as functions of pressure and compared with the calculated values in Figs. 2(a) and 2(b), respectively. Experiment and theory agree closely for this structure. The transition from $\mathrm{Cmcm}$ to the $P 2_{1} / \mathrm{m}$ structure involves shearing of the (100) plane relative to the neighboring plane (Fig. 3). The (100) plane is a pseudohexagonal plane on which $\mathrm{Cd}$ and $\mathrm{Te}$ atoms occupy the sites alternatively in a zigzag manner. The neighboring (100) plane has identical geometry but the site occupations are alternated. As such, the closest Cd-Te distance occurs between adjacent (100) planes, connecting the $\mathrm{Cd}$ and $\mathrm{Te}$ on top of each other. During the transition, the (100) planes become puckered and shift relative to the neighboring planes in the [010] direction. After the phase transition, these planes become the (001) planes in the $P 2_{1} / m$ structure (Fig. 3). If the transition continued along this path, it would eventually reach a high-symmetry $B 11(P 4 / \mathrm{nmm})$ structure, the structure of $\gamma$-CuTi. Specifically, for the $P 2_{1} / m$ structure to become the $B 11$ structure, the $\mathrm{Cd} 2 e$ sites need to change to $(0.5,0.25,0.3423)$ and $(1.0,0.25,0.6578)$ and the Te $2 e$ sites change to $(0.0,0.25,0.1048)$ and $(0.5,0.25,0.8952)$, while the lattice parameters change to $a=5.01, b=5.01$, and $c=5.71 \AA$. Moreover, the XRD pattern of the $P 2_{1} / m$ structure shows a resemblance to that of the $B 11$ structure, whereas the peak broadening due to the distortion is obvious [Fig. 4(a)]. From this analysis, it seems appropriate to suggest the $P 2_{1} / \mathrm{m}$ structure as an intermediate between the $\mathrm{Cmcm}$ and

TABLE I. Experimental and calculated structural parameters of rocksalt, $\mathrm{Cmcm}$, and post $-\mathrm{Cmcm}\left(P 2_{1} / \mathrm{m}\right)$ phases of CdTe at selected pressures: space group (SG), number of formula units in the unit cell $Z$, lattice parameters, cell volume, bulk modulus $B$ and its pressure derivative $B^{\prime}$, Wyckoff site, and the corresponding coordinates. Calculated values are presented under the experimental values.

\begin{tabular}{|c|c|c|c|c|c|c|c|c|c|c|c|c|}
\hline$P(\mathrm{GPa})$ & SG & $Z$ & $a(\AA)$ & $b(\AA)$ & $c(\AA)$ & $V\left(\AA^{3}\right)$ & $B(\mathrm{GPa})$ & $B^{\prime}$ & WP & $x$ & $y$ & $z$ \\
\hline 6 & $F m-3 m$ & 4 & $\begin{array}{l}5.861(1) \\
5.869\end{array}$ & $\begin{array}{l}5.861(1) \\
5.869\end{array}$ & $\begin{array}{l}5.861(1) \\
5.869\end{array}$ & $\begin{array}{l}201.67(5) \\
202.262\end{array}$ & $77.4(8)$ & 4 (fixed) & $\begin{array}{l}\mathrm{Cd}(4 a) \\
\operatorname{Te}(4 c)\end{array}$ & $\begin{array}{r}0 \\
0.25\end{array}$ & $\begin{array}{r}0 \\
0.25\end{array}$ & $\begin{array}{c}0 \\
25\end{array}$ \\
\hline 30 & $\mathrm{Cmcm}$ & 4 & $\begin{array}{l}5.454(5) \\
5.461\end{array}$ & $\begin{array}{l}5.850(5) \\
5.903\end{array}$ & $\begin{array}{l}5.122(5) \\
5.036\end{array}$ & $\begin{array}{l}163.36(3) \\
162.34\end{array}$ & $95.6(9)$ & $3.2(6)$ & $\begin{array}{l}\mathrm{Cd}(4 c) \\
\mathrm{Te}(4 c)\end{array}$ & $\begin{array}{l}0 \\
0 \\
0 \\
0\end{array}$ & $\begin{array}{l}0.644(4) \\
0.633 \\
0.192(4) \\
0.165\end{array}$ & $\begin{array}{l}0.25 \\
0.25 \\
0.25 \\
0.25\end{array}$ \\
\hline 63 & $P 2_{1} / m$ & 4 & $\begin{array}{l}5.823(5) \\
5.879\end{array}$ & $\begin{array}{l}4.728(7) \\
4.741\end{array}$ & $\begin{array}{l}4.906(8) \\
4.900\end{array}$ & $\begin{array}{l}135.06(6) \\
136.607\end{array}$ & $220(4)$ & 4 (fixed) & $\begin{array}{l}\mathrm{Cd}(2 e) \\
\mathrm{Cd}(2 e) \\
\mathrm{Te}(2 e) \\
\operatorname{Te}(2 e)\end{array}$ & $\begin{array}{l}0.5051 \\
0.8025 \\
0.0054 \\
0.3281\end{array}$ & $\begin{array}{l}0.25 \\
0.25 \\
0.25 \\
0.25\end{array}$ & $\begin{array}{l}0.3150 \\
0.7820 \\
0.2779\end{array}$ \\
\hline
\end{tabular}



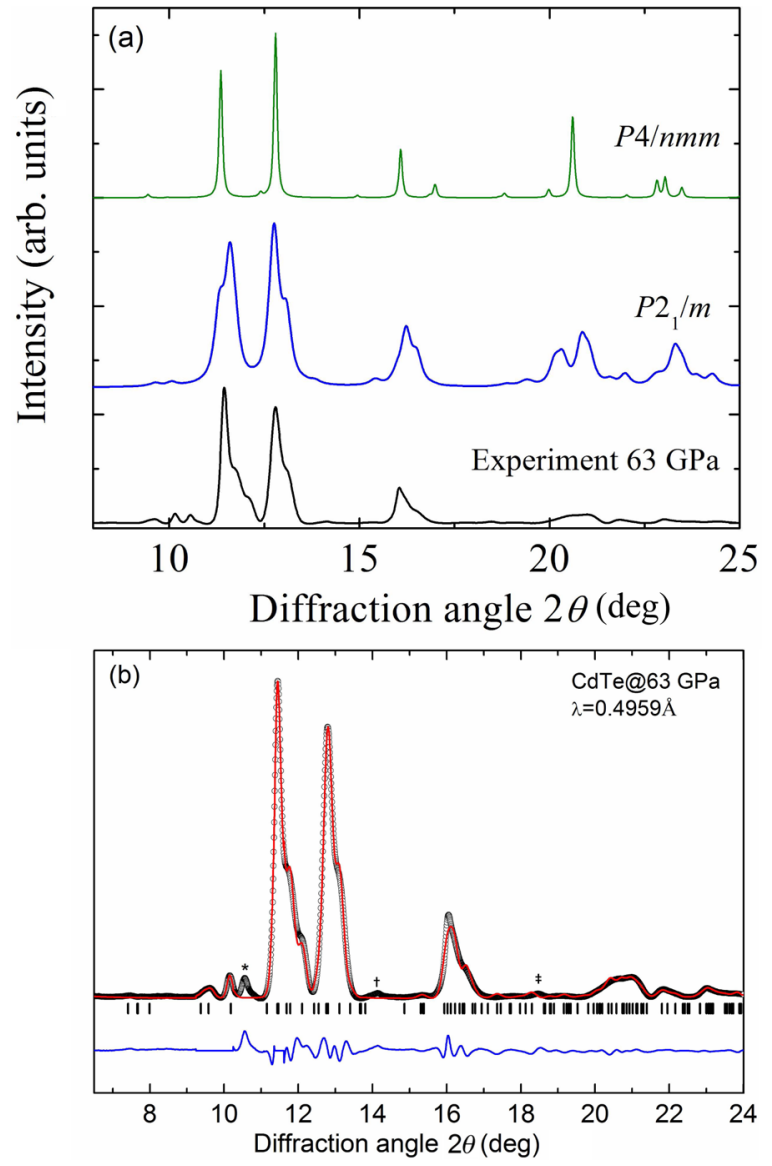

FIG. 4. (a) Experimental XRD pattern for the post- $\mathrm{Cmcm}$ phase at $63 \mathrm{GPa}$ compared with the simulated XRD pattern for the $P 2{ }_{1} / \mathrm{m}$ and $P 4 / \mathrm{nmm}$ structures at the same pressure and (b) Le Bail refinement results for the $P 2_{1} / \mathrm{m}$ structure of CdTe at $63 \mathrm{GPa}$. Symbols correspond to the measured profile and the red solid lines represent the results of Le Bail refinement. The difference curves (blue curves) are shown also. Vertical ticks mark the positions of the Bragg peaks of the of the $P 2_{1} / \mathrm{m}$ phase of CdTe. The dagger and double dagger mark the locations of Bragg peaks due to $\mathrm{Re}$ (gasket) and $\mathrm{Ne}$ (PTM), respectively. The asterisk marks a Bragg peak that cannot be explained at the moment.

$B 11$ structures and the common subgroup for this transition path. In a broader pressure range, the phase transition of $\mathrm{CdTe}$ is viewed as a $B 1$ to $B 11$ transition by passing $C m c m$ and $P 2_{1} / m$ intermediate phases (Fig. 3).

To examine the energetics, the enthalpies of the $\mathrm{Cmcm}$, $P 2_{1} / m$, and $B 11$ structures were calculated at different pressures (Fig. 5). This calculation shows that the $P 2_{1} / m$ structure becomes thermodynamically more stable than the $\mathrm{Cmcm}$ structure near $45 \mathrm{GPa}$, indeed very close to the experimental transition pressure (46 GPa). The $B 11$ structure is calculated to be more stable than the $P 2_{1} / m$ structure near $68 \mathrm{GPa}$. This finding establishes the $P 2_{1} / m$ structure as the intermediate phase between the $\mathrm{Cmcm}$ and $\mathrm{P} 4 / \mathrm{nmm}$ structures. Previously, the $B 2$ structure (as in $\mathrm{CsCl}$ ) was suggested as a candidate structure for the post- $\mathrm{Cmcm}$ phase on account of its low Madelung energy, which becomes dominant in determining structures at small volumes [30,31]. After all, the $B 1$ to $B 2$ transition has been found in many covalent binaries under

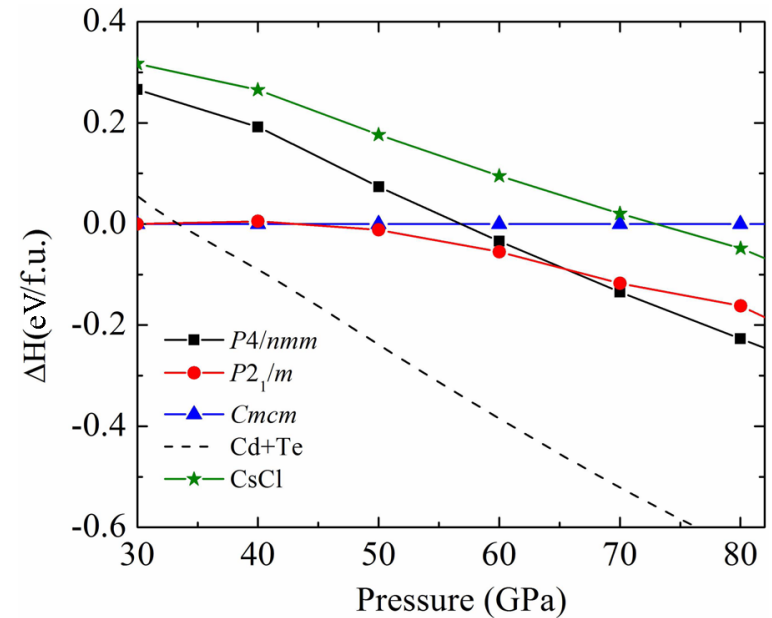

FIG. 5. Calculated enthalpies for $C m c m, P 2_{1} / m, P 4 / n m m$, and $B 2(\mathrm{CsCl})$ structures at different pressures. The enthalpy of the $\mathrm{Cmcm}$ structure is used as the zero-energy reference. The enthalpy sum for elemental solids $\mathrm{Cd}$ and $\mathrm{Te}$ is present for comparison.

high pressure. Our calculation shows that the $B 2$ structure of CdTe indeed becomes more stable than the $\mathrm{Cmcm}$ structure near $73 \mathrm{GPa}$, but is never more stable than the $P 2_{1} / m$ and $B 11$ structures. The $B 2$ structure therefore does not have a region of stability. In terms of the electronic structure, all four structures were found to be metallic from electronic band and density of states calculations. This agrees with previous observations that $\mathrm{CdTe}$ turns metallic in the $B 1$ structure.

The calculated transition sequence in Fig. 5 agrees very well with the experiment. However, an intriguing finding puzzles us, that is, the enthalpy of CdTe is higher than the enthalpy sum of its elemental constituents $(\mathrm{Cd}$ and $\mathrm{Te})$ at pressures higher than $34 \mathrm{GPa}$. In fact, this unusual behavior of CdTe has been pointed out in a previous DFT study [9]. From a thermodynamic perspective, this suggests that $\mathrm{CdTe}$ is prone to decomposition, but this obviously contradicts the fact that CdTe is stable to at least $63 \mathrm{GPa}$. A comparison of the experimental patterns of CdTe above $34 \mathrm{GPa}$ with the calculated patterns of $\mathrm{Cd}(\mathrm{hcp})$ and $\mathrm{Te}$ (bcc) at the same pressures [26,27] definitely rules out the possibility of a decomposition. Interestingly, a similar situation has been found in $\mathrm{ZnTe}$ as well, that $\mathrm{ZnTe}$ is calculated to dissociate to elemental components at $38 \mathrm{GPa}$ [32], but experiment shows that it is stable to at least up to $85 \mathrm{GPa}$ [10]. Although this is somewhat mysterious, it may not be a coincidence but something related to tellurium compounds. To this end, the mechanical and dynamical stabilities of the $P 2_{1} / m$ and $B 11$ structures are examined by phonon calculations [Figs. 6(a) and 6(b)]. The absence of the imaginary frequencies in the phonon dispersion confirms that these two structures are dynamically stable. The established dynamical stability ensures that these two structures do not decompose into elements. Thus, the $P 2_{1} / \mathrm{m}$ and $B 11$ phases are metastable high-enthalpy phases.

One contributing factor to this discrepancy is the temperature effects. Our calculations are for the ground states at $T=0 \mathrm{~K}$, so they likely underestimate or overestimate the decomposition pressure, but the errors should be minor since the vibrational free energy is only on the order of 

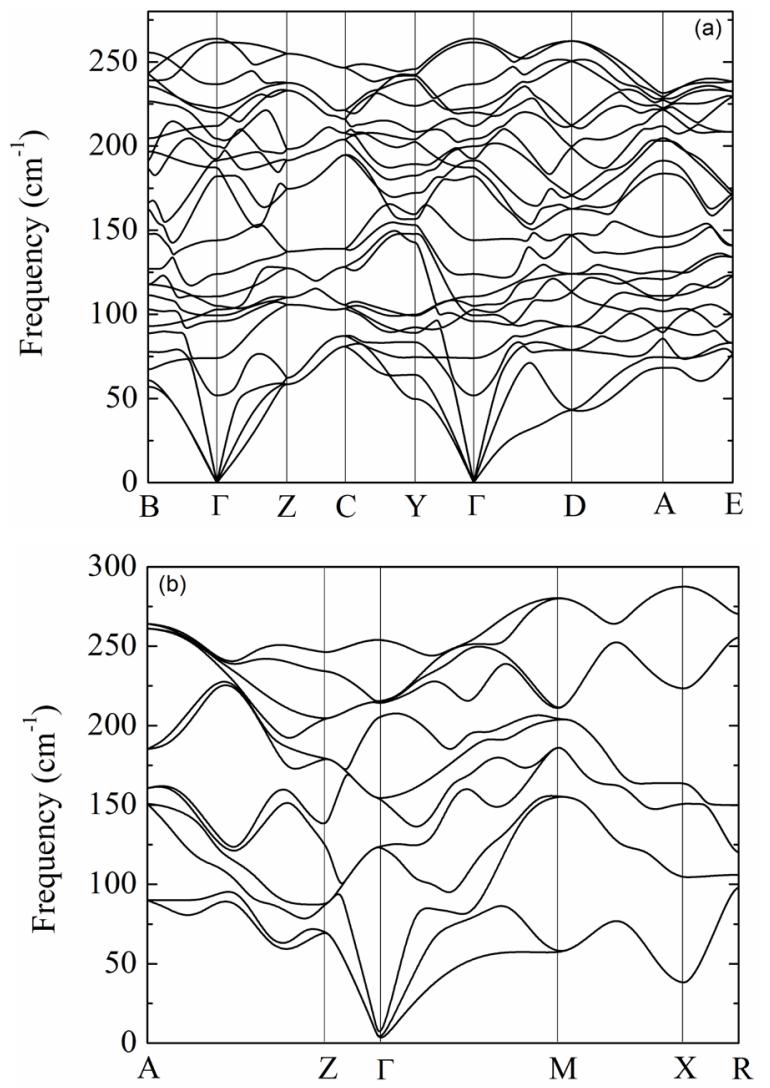

FIG. 6. Calculated phonon dispersion relations for (a) the $P 2_{1} / m$ structure at $60 \mathrm{GPa}$ and (b) the $P 4 / \mathrm{nmm}$ structure at $80 \mathrm{GPa}$.

$10 \mathrm{meV} /$ atom at room temperature. Moreover, since CdTe, $\mathrm{Cd}$, and $\mathrm{Te}$ are all in the solid state at room temperature, there are no large heat reservoirs, i.e., heat of fusion and vaporization, to reverse the huge enthalpy difference between CdTe and the elements. Thus, we speculate that there exists a large kinetic energy barrier for the decomposition of CdTe, which necessitates an activation energy (such as extra pressure or elevated temperature) to overcome it. For example, the high-pressure synthesis of new compounds from elemental constituents, which is the reverse process of decomposition, always requires much higher pressure than what is predicted for the dissociation pressure and most of the time requires increased temperature [33-35].

To gain deeper insight into the stability of CdTe at elevated pressures in Fig. 2(b) we compare the atomic volume of CdTe with the experimentally determined $[26,27]$ and the calculated (in this study) volume of the superposition of $(\mathrm{Cd}+\mathrm{Te}) / 2$ as a function of pressure. As can be clearly seen, the atomic volume of CdTe is always larger than the superposition of $(\mathrm{Cd}+\mathrm{Te}) / 2$ both below and above the critical pressure for decomposition ( $34 \mathrm{GPa}$ ) as determined by enthalpy calculations. In fact, the atomic volume of CdTe is always larger than that of its constituents even at ambient conditions [36]. This observation implies that CdTe has a much lower potential energy (higher stability) than the superposition of $(\mathrm{Cd}+\mathrm{Te}) / 2$ and provides a plausible explanation for the experimentally observed stability of CdTe at higher pressures. Still, the observed stability of CdTe for more than $30 \mathrm{GPa}$ above the theoretical dissociation point is very significant, and future investigation is encouraged to address this interesting question.

\section{SUMMARY}

The high-pressure phase transition of CdTe has been investigated by a combined experimental and computational study up to $63 \mathrm{GPa}$. The previously reported $B 1$ to $\mathrm{Cmcm}$ and $\mathrm{Cmcm}$ to post- $\mathrm{Cmcm}$ phase transitions were successfully identified by synchrotron XRD measurements. The long-sought crystal structure for the post-Cmcm phase was characterized as a monoclinic $P 2_{1} / m$ structure. Analysis of the phase transition pathway suggested that the $P 2_{1} / m$ structure is an intermediate phase between the $C m c m$ and $B 11$ phases. Using enthalpy calculation, the $P 2_{1} / m$ to $B 11$ phase transition was predicted to occur near $68 \mathrm{GPa}$. In the pressure range of interest, the enthalpy of CdTe is constantly higher than the enthalpy sum of $\mathrm{Cd}$ and Te, indicating that $\mathrm{CdTe}$ is a high-enthalpy compound which is stabilized by a tremendous kinetic barrier.

\section{ACKNOWLEDGMENTS}

This work was supported by DARPA (Grants No. W31P4Q1310005 and No.W31P4Q1210008), the Deep Carbon Observatory, and Natural Sciences and Engineering Research Council of Canada. Part of this work was performed under the auspices of the U.S. Department of Energy by Lawrence Livermore National Security, LLC under Contract No. DE-AC52-07NA27344. The ALS is supported by the Director, Office of Science, BES of DOE under Contracts No. DE-AC02-05CH11231 and No. DE-AC02-06CH11357. The authors thank Andrew Doran for helping with the gas loading at Beamline 12.2.2. Computing resources were provided by the University of Saskatchewan, Westgrid, and Compute Canada.
[1] J. Mei and V. Lemos, Solid State Commun. 52, 785 (1984).

[2] A. N. Chantis, M. van Schilfgaarde, and T. Kotani, Phys. Rev. Lett. 96, 086405 (2006).

[3] M. Powalla, S. Paetel, E. Ahlswede, R. Wuerz, C. D. Wessendorf, and T. Magorian Friedlmeier, Appl. Phys. Rev. 5, 041602 (2018).

[4] R. Weil, R. Nkum, E. Muranevich, and L. Benguigui, Phys. Rev. Lett. 62, 2744 (1989).
[5] Y. Pei, A. D. LaLonde, N. A. Heinz, and G. J. Snyder, Adv. Energy Mater. 2, 670 (2012).

[6] S. M. Reimann and M. Manninen, Rev. Mod. Phys. 74, 1283 (2002).

[7] R. J. Nelmes, M. I. McMahon, N. G. Wright, and D. R. Allan, Phys. Rev. B 48, 1314 (1993).

[8] R. J. Nelmes, M. I. McMahon, N. G. Wright, and D. R. Allan, Phys. Rev. B 51, 15723 (1995). 
[9] Y. Yan, S. Zhang, Y. Wang, G. Yang, and Y. Ma, RSC Adv. 5, 104426 (2015).

[10] R. Nelmes and M. McMahon, in High Pressure Semiconductor Physics I, edited by T. Suski and W. Paul, Semiconductors and Semimetals Vol. 54 (Elsevier, Amsterdam, 1998), pp. 145-246.

[11] S. Biering and P. Schwerdtfeger, J. Chem. Phys. 137, 034705 (2012).

[12] M. Kunz, A. MacDowell, W. Caldwell, D. Cambie, R. Celestre, E. Domning, R. Duarte, A. Gleason, J. Glossinger, N. Kelez, D. Plate, T. Yu, J. Zaug, H. Padmore, R. Jeanloz, A. Alivisatos, and S. Clark, J. Synchrotron Radiat. 12, 650 (2005).

[13] M. Matsui, J. Phys.: Conf. Ser. 215, 012197 (2010).

[14] K. Syassen, High Press. Res. 28, 75 (2008).

[15] C. Prescher and V. B. Prakapenka, High Press. Res. 35, 223 (2015).

[16] W. Kraus and G. Nolze, J. Appl. Crystallogr. 29, 301 (1996).

[17] A. C. Larson and R. B. V. Dreele, GSAS: General Structure Analysis System, Los Alamos National Laboratory Report No. LAUR 86-748, 2000.

[18] A. Boultif and D. Louër, J. Appl. Crystallogr. 37, 724 (2004).

[19] R. Martoňák, A. Laio, and M. Parrinello, Phys. Rev. Lett. 90, 075503 (2003).

[20] G. Kresse and D. Joubert, Phys. Rev. B 59, 1758 (1999).

[21] P. E. Blöchl, Phys. Rev. B 50, 17953 (1994).

[22] G. Kresse and J. Furthmüller, Comput. Mater. Sci. 6, 15 (1996).

[23] J. P. Perdew, K. Burke, and M. Ernzerhof, Phys. Rev. Lett. 77, 3865 (1996).
[24] P. Giannozzi, S. Baroni, N. Bonini, M. Calandra, R. Car, C. Cavazzoni, D. Ceresoli, G. L. Chiarotti, M. Cococcioni, I. Dabo et al., J. Phys.: Condens. Matter 21, 395502 (2009).

[25] F. Birch, J. Geophys. Res. 83, 1257 (1978).

[26] K. Takemura, Phys. Rev. B 56, 5170 (1997).

[27] G. Parthasarathy and W. B. Holzapfel, Phys. Rev. B 37, 8499 (1988).

[28] J. Z. Hu, Solid State Commun. 63, 471 (1987).

[29] T.-L. Huang and A. L. Ruoff, Phys. Rev. B 31, 5976 (1985).

[30] N. Benkhettou, D. Rached, and M. Rabah, Czech. J. Phys. 56, 409 (2006).

[31] R. Ahuja, P. James, O. Eriksson, J. M. Wills, and B. Johansson, Phys. Status Solidi B 199, 75 (1997).

[32] Z. Li, H. Wang, Y. Li, Y. Ma, T. Cui, and G. Zou, New J. Phys. 12, 043058 (2010).

[33] E. Stavrou, Y. Yao, A. F. Goncharov, S. S. Lobanov, J. M. Zaug, H. Liu, E. Greenberg, and V. B. Prakapenka, Phys. Rev. Lett. 120, 096001 (2018).

[34] B. A. Steele, E. Stavrou, J. C. Crowhurst, J. M. Zaug, V. B. Prakapenka, and I. I. Oleynik, Chem. Mater. 29, 735 (2017).

[35] A. Adeleke, M. Kunz, E. Greenberg, V. B. Prakapenka, Y. Yao, and E. Stavrou, ACS Earth Space Chem. 3, 2517 (2019).

[36] A. Jain, S. P. Ong, G. Hautier, W. Chen, W. D. Richards, S. Dacek, S. Cholia, D. Gunter, D. Skinner, G. Ceder, and K. A. Persson, APL Mater. 1, 011002 (2013). 